\title{
Prevention and Management of Intraoperative Floppy Iris Syndrome
}

\author{
James A. Davison \\ Wolfe Eye Clinic, Iowa, \\ USA
}

\section{Introduction}

Other than using it to attach the IOL optic in McCannel suture procedures, the iris, in any amount, only causes problems for the cataract surgeon. In its extreme, there are no iris related complications in aniridia patients, other than not having an iris. Even in normal cases, there are always some disturbances of iris tissue as evidenced by postoperative inflammation, pigment dispersion, and sometimes iris transillumination.

One of the keys then to successful cataract surgery is to reduce the effect that the iris has on the intra and postoperative course of events. So during cataract surgery, the iris needs to be minimized and isolated. We dilate the pupil as much as we can which gives us better visualization and less iris to influence either directly by touching or indirectly through hydraulic means. The key is to make the iris as inconsequential as it can be by reducing its area pharmacologically or physically. If the iris area presentation is large to start with, there will be more of it to be caught in the flow of BSS and it may become floppy in the fluidic turbulence. If it is dilated and contracted, there will be less presentation of tissue to BSS flow and less muscle reaction to the buffeting of turbulence.

\section{Planning}

The axiom "the pupil never gets larger during surgery" needs to be a critical part of the surgery planning process. Whatever the pupil diameter is at the start of a case, it will only stay the same or get smaller as the case goes on. We have a good estimate of surgical pupil size when we see patients in the office so we can plan ahead. Sometimes the pupil gets larger before surgery vs. the clinic because of the stronger drops used in surgery rather than in the examination lane. My general rule is that I want at least a $4.5-5.0 \mathrm{~mm}$. pupil at the outset of surgery so that I can get an additional $0.5 \mathrm{~mm}$. dilation with viscoelastic anterior chamber pressurization and I can create the tremendously important capsulorhexis in a diameter of approximately $5.0-5.5 \mathrm{~mm}$. in ideal circumstances but at least $4.5 \mathrm{~mm}$. at a minimum. But clinical circumstances may appear to require an even larger pupil because of lens and/or zonule character. So a $4.5 \mathrm{~mm}$. pupil in a normal eye with an average character cataract of LOCS III NO 3.7 NC 3.7, (Chylack 1993) will likely be maintained because surgery should be routine, brief, and uneventful. But that same pupil size may not be 
maintainable during a longer more difficult case such as if the cataract is very soft, very hard, or if it is accompanied by an inherently loose zonule as in pseudoexfoliation, or if the iris is unusually flaccid. A pupil of $6.0 \mathrm{~mm}$. is desirable at the outset of these types of difficult cases.

If the pupil is smaller than $4.5 \mathrm{~mm}$. and I can't create a capsulorhexis of that diameter, I employ a mechanical device to physically enlarge it prior to performing capsulorhexis. That rule has yielded an overall rate of mechanical pupil enlargement for me of $10 \%$ but an iris related complication rate (significant iris aspiration or prolapse or an associated posterior capsule rupture) of zero (unpublished data, JAD 2008-2011). This utilization may seem high, but the risks of problems and the significance of the problems created by a small pupil are more important to the outcome of these cases than the time and expense it takes to temporarily physically enlarge the pupil and accomplish them in normal fashion

\section{Pharmacologic pupil dilation}

A topical non-steroidal preparation is used 3 days prior to surgery in the hopes of reducing inflammation and maybe assisting in dilating the pupil. There are numerous ways to accomplish the instillation but, Cyclogyl 1\% and NeoSynephrine 2.5\% are longtime key ingredients for dilating the eye starting about 45 minutes prior to surgery. Lidocaine $1 \%$ preservative free is injected into the anterior chamber just after the normal clear corneal primary and paracentesis incisions are created. This substance also assists with initiating and maintaining pupil dilation. Epinephrine 1:10,000 (1 ml.) is used in $500 \mathrm{cc}$ in the continuous irrigation of BSS

Epi-Shugarcaine (Shugar 2006), a mixture of preservative free lidocaine and epinephrine has been shown by Joel Shugar, MD to be an effective supplementary agent in maintaining pupil dilation just before phacoemulsification and again before cortex aspiration as well. Intraoperative unpreserved epinephrine preceded by topical atropine has also been demonstrated to achieve and maintain larger pupil sizes and help reduce the incidence of IFIS (Masket 2007).

\section{Mechanical pupil dilation}

Viscoelastic anterior chamber pressurization prior to performing capsulorhexis will generally yield $0.5 \mathrm{~mm}$. diameter additional pupil dilation. I use a DuoVisc (Alcon Surgical) strategy injecting enough Viscoat (Alcon Surgical) to fill approximately $40 \%$ of the anterior chamber and following with ProVisc (Alcon Surgical) underneath to the point that some is just refluxing out the incision as it's being injected. If I can achieve a $4.5 \mathrm{~mm}$ pupil, I'll generally proceed with capsulorhexis but if I can't I will mechanically dilate the pupil with one of two devices.

I employ the Graether Pupil Expander (EagleVision) which was invented by John Graether, MD in 1991 (Graether 1996) and use it part of the time i.e. in normal eyes with small pupils. Its advantages are the average time it takes to accomplish the insertion, about 30 - 60 seconds, removal in about 10 seconds, and the fact that no additional incisions are needed. 


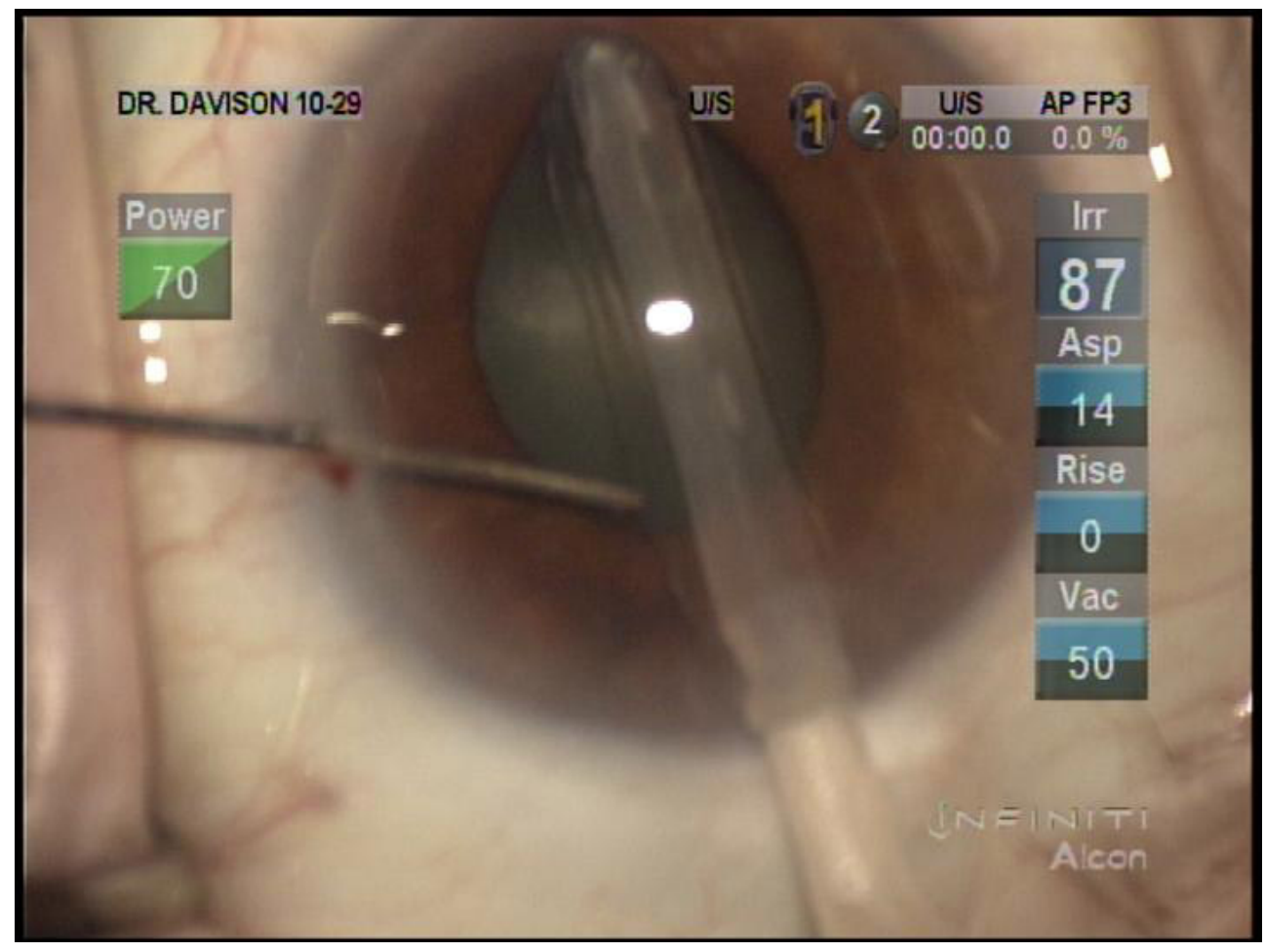

Fig. 1. The expander is draped over its insertion rod and has engaged the distal pupillary iris 


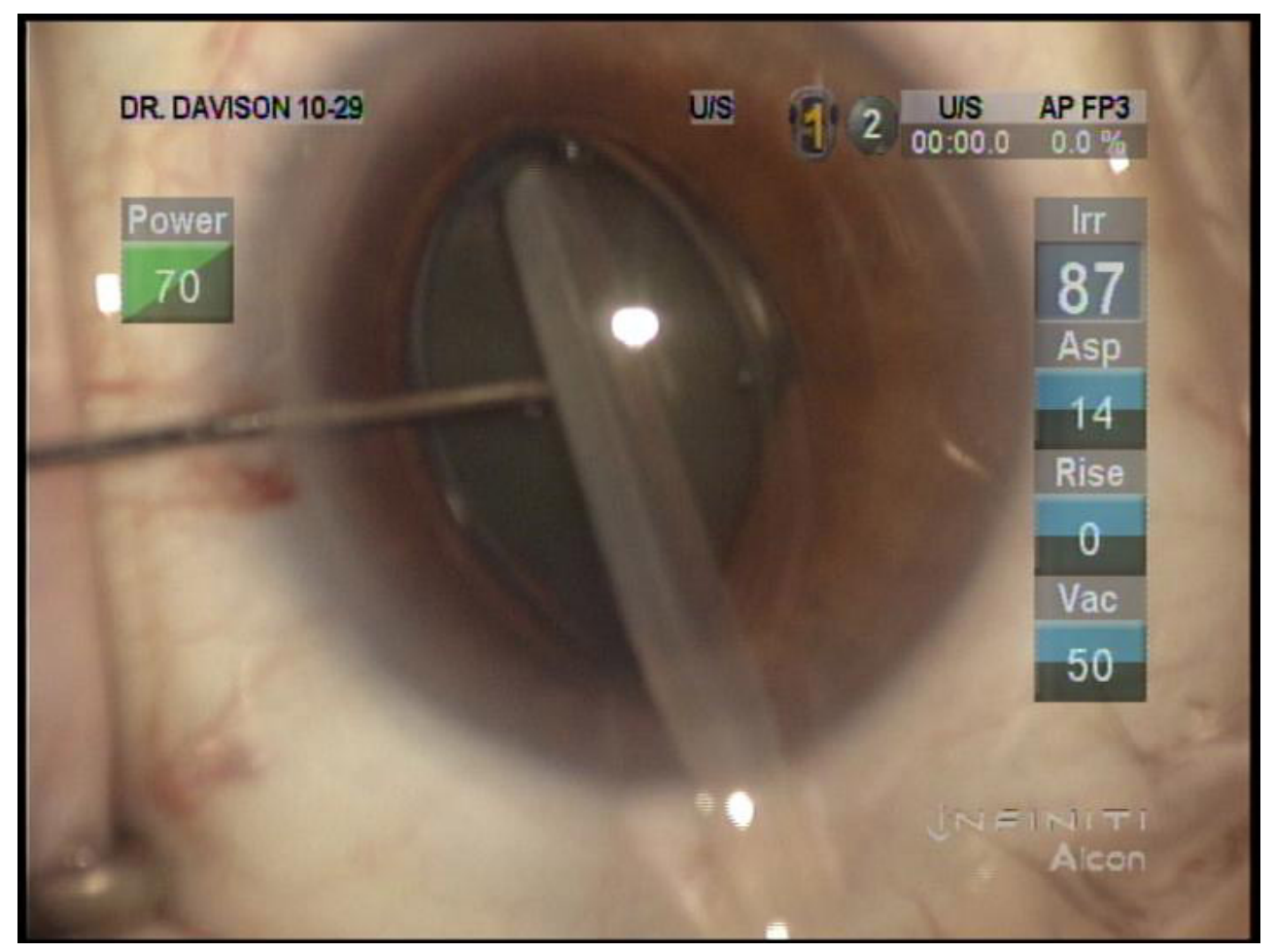

Fig. 2. As the insertion rod is withdrawn, the expander is engaged distally and on the left side but has missed engaging the pupil on the right side. 


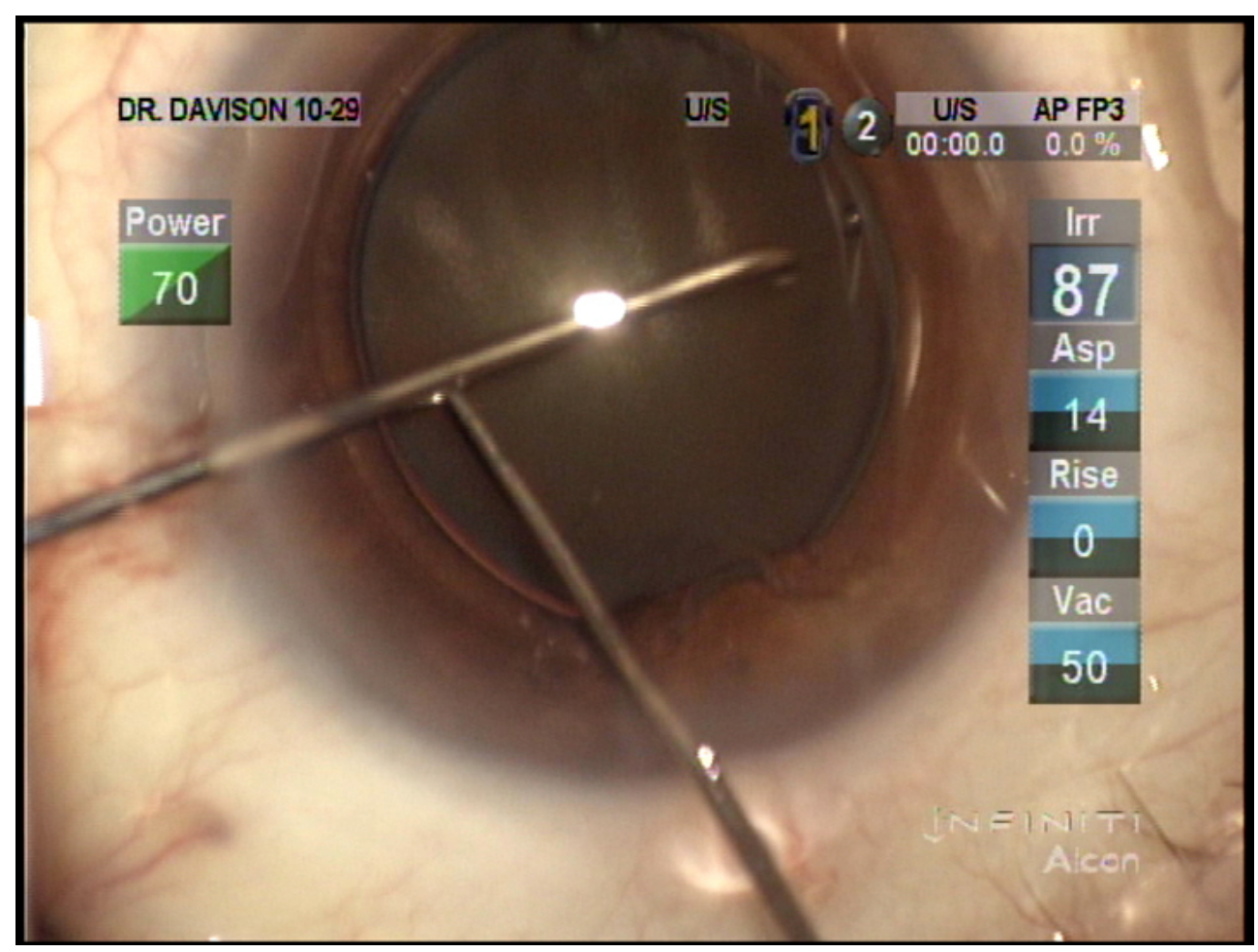

Fig. 3. The Lester hook and side port manipulator have just completed a kind of cross handed stretching maneuver and are about to be withdrawn. The expander is fully engaged and the pupil size will be maintained throughout the entire case. The Lester hook will engage the small proximal strap and easily withdraw the expander at the conclusion of the case

Interestingly, it is easier to insert in smaller pupils rather than medium sized ones because the pupillary iris tissue more tightly grabs the attachment points if the pupil is smaller rather than if it is larger where it tends to fall off. But, I worry that, at least in my hands, the downward pressure required to place the device may have a tendency to create some zonular fiber disruption in some cases. This has never been clinically significant to me but I still tend not to use it in patients with pseudoexfoliation or trauma cases which have an already loose zonule propensity.

In those patients with pseudoexfoliation and a potentially weak zonule, medium size pupils or eyes with very compact anterior segment anatomy, I generally employ Grieshaber iris retractors (Alcon Surgical). Their disadvantage is that they require 4 additional incisions and take about 2 minutes to place and maybe 30 seconds to remove. I orient them in a square pattern by creating incisions with a 22,5 degree blade. I create all four of the incisions first, and then create the paracentesis incision for my left hand and $2.4 \mathrm{~mm}$ keratome primary incision before introducing intracameral lidocaine or viscoelastic. 


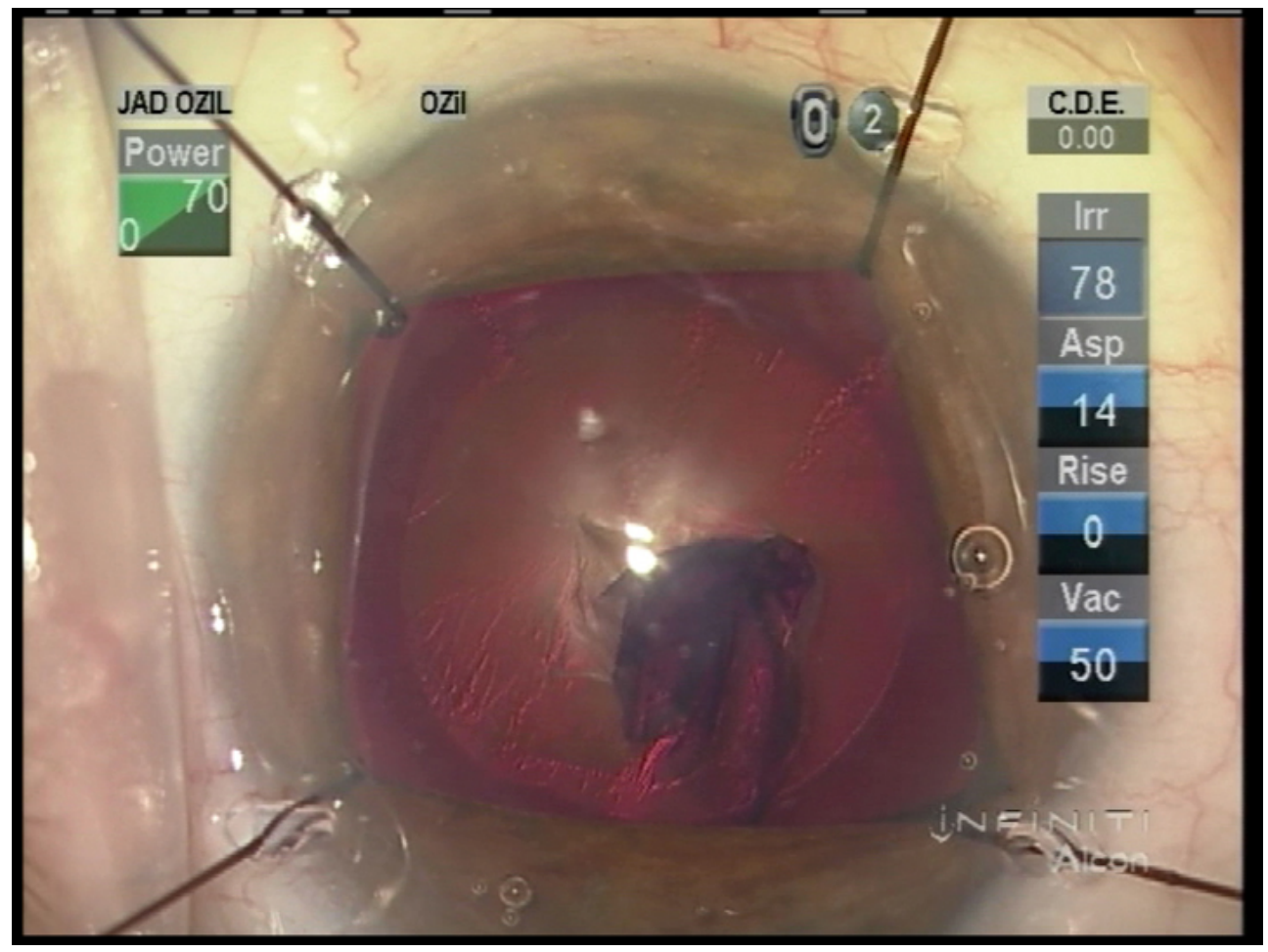

Fig. 4. The capsulorhexis has just been completed using Trypan blue dye. The retractors have been placed in each quadrant and fairly fully retracted to expose the anterior capsule for CCC creation and hydrodissection.

Prior to introducing the phacoemulsification tip, I loosen the proximal retractors so that the iris falls posterior and will not be rubbed by the phaco tip and sleeve during emulsification of the lens. 


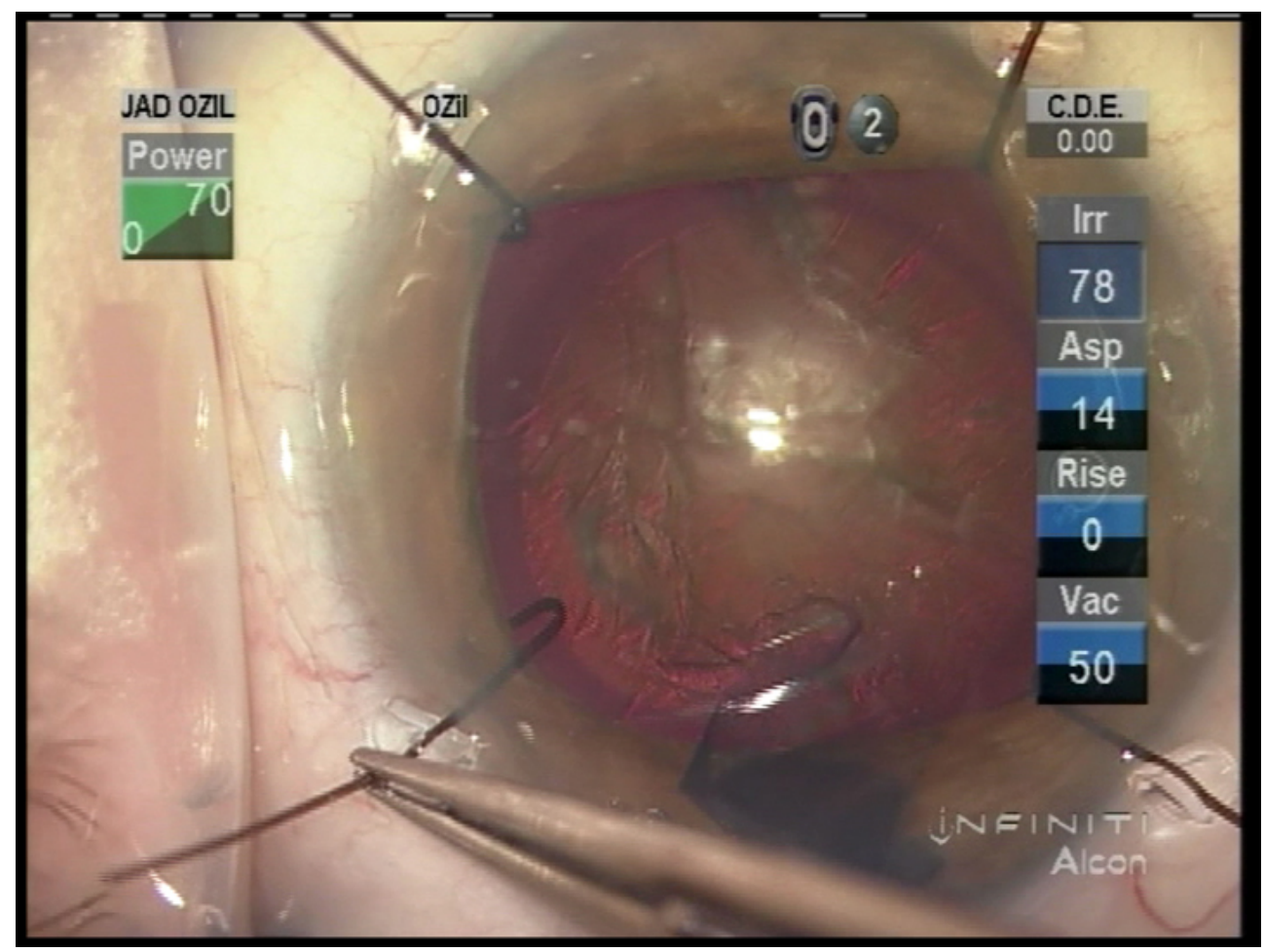

Fig. 5. The retractor on the lower left is being pushed into the anterior chamber with a tying forceps for a length of about $1.0 \mathrm{~mm}$. The iris will hug the retractor in its new position within a second just as it already has the retractor on the lower right. Just that much less retraction under the phacoemulsification tip allows the iris to fall away from it and not suffer damage from its vibration.

\section{Physically dampening hydraulic shock and minimizing turbulence}

During the sculpting phase of contemporary in situ fracture phacoemulsification there is a balanced and continuous inflow and outflow of BSS. This equilibrium exists because of the advantages of modern cataract surgery equipment and technique. Only a small volume controlled leakage of BSS is observed to leak around the infusion sleeve. A similar amount of leakage occurs during the quadrant removal phase of the surgery as well. Unnecessary surgeon generated motions are eliminated as are extremes and consequent substantial changes in vacuum and occlusion levels.

Most machines can create this scenario to varying degrees. I use the Alcon Infiniti (Alcon Surgical) micro-coaxial system. In this system, all of the components are matched to each other in order to minimize imprecision and maximize efficiency, reproducibility, and safety. The $2.4 \mathrm{~mm}$. keratome is capable of creating a perfect $2.4 \mathrm{~mm}$. width with a $1.8 \mathrm{~mm}$. length shelf incision before the introduction of viscoelastic. This can be accomplished while the 22.5 degree angle paracentesis blade stabilizes the globe. 


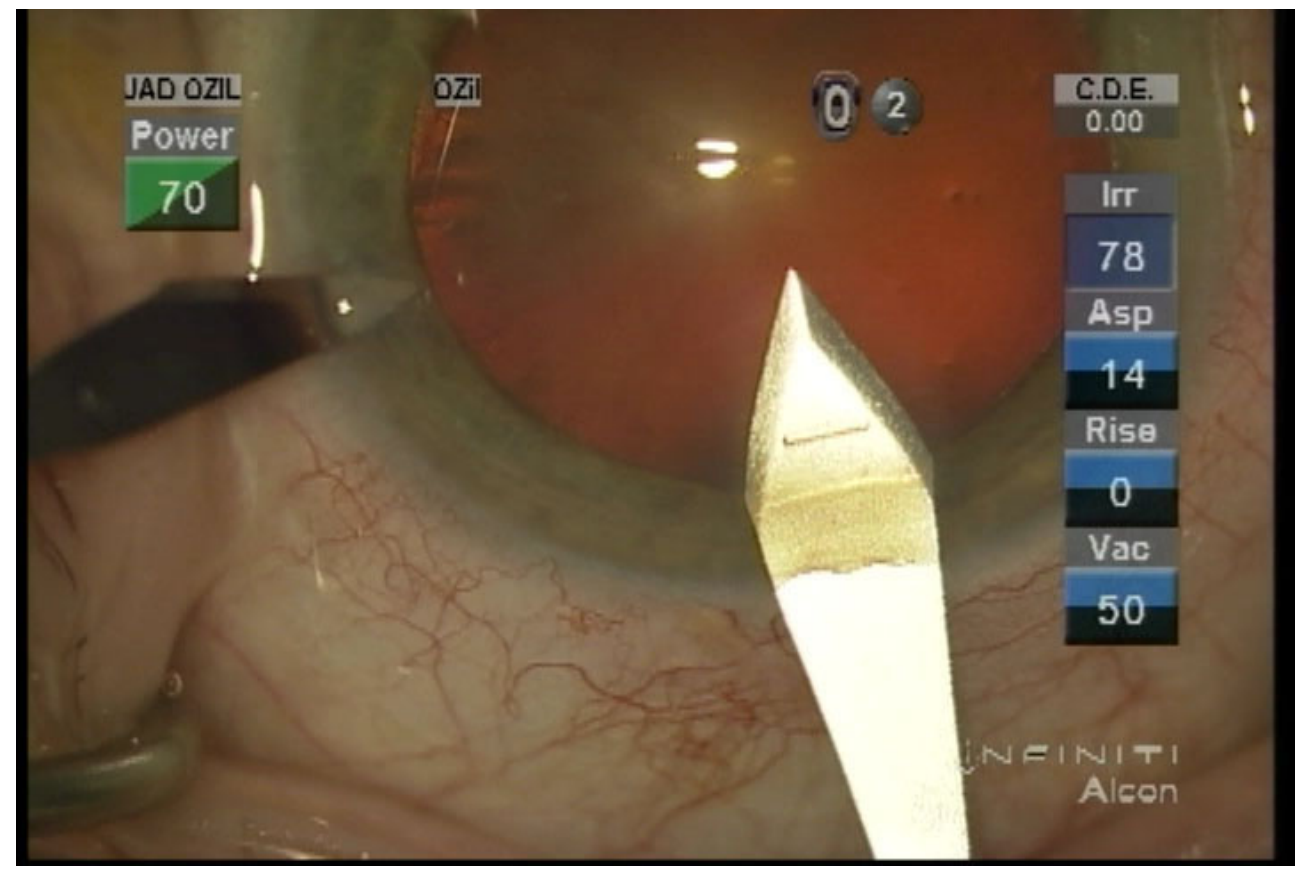

Fig. 6 . The 22.5 degree angle paracentesis blade stabilizes the globe by pulled slightly upward and toward the surgeon while the $2.4 \mathrm{~mm}$ keratome is used to create the primary incision.)

The Ultra infusion sleeve is specifically designed to use within that incision surrounding the $0.9 \mathrm{~mm}$. outside diameter shaft phacoemulsification tip (12 degree shaft bend mini-flare, 45 degree aperture angle, ABS featured). 


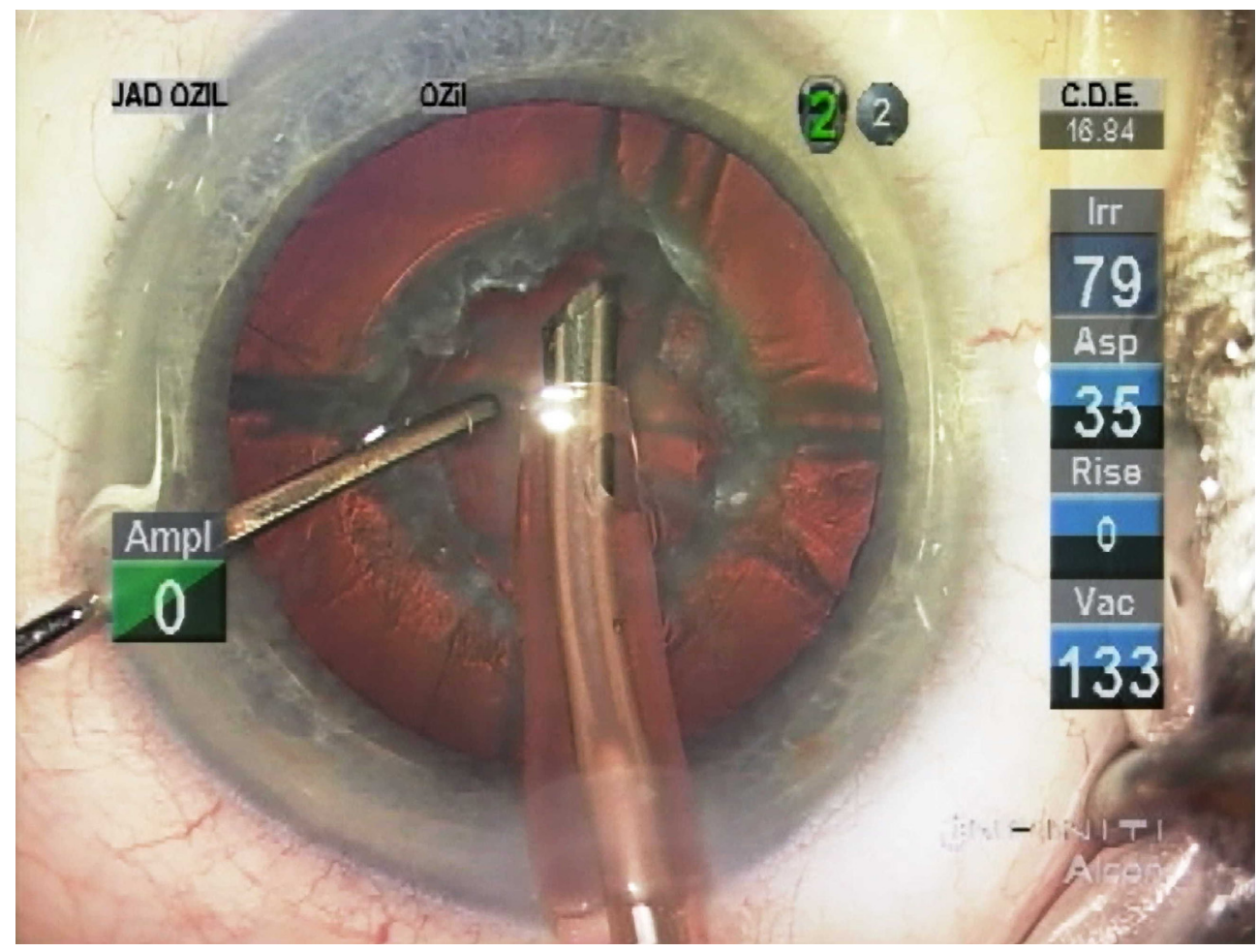

Fig. 7. The $0.9 \mathrm{~mm}$. outside diameter 12 degree shaft bend tip is surrounded by the Ultra infusion sleeve through a $2.4 \mathrm{~mm}$. clear corneal incision in the micro-coaxial system.

For sculpting, I use relatively low settings featuring a bottle height of $78 \mathrm{cms}$, surgeon control of continuous longitudinal phacoemulsification maximum $90 \%$, vacuum of 50 mmHg., and AFR of $14 \mathrm{cc}$./min. I use enough power as necessary to efficiently sculpt to a level without displacing the lens that will create circumstances that yield four equal nuclear fragments after cracking. If the lens is hard, I'll use the tip on its side in sculpt mode to reduce the size of the quadrants by buzzing off their internal corners thus rendering them a relatively two-dimensional plate. 


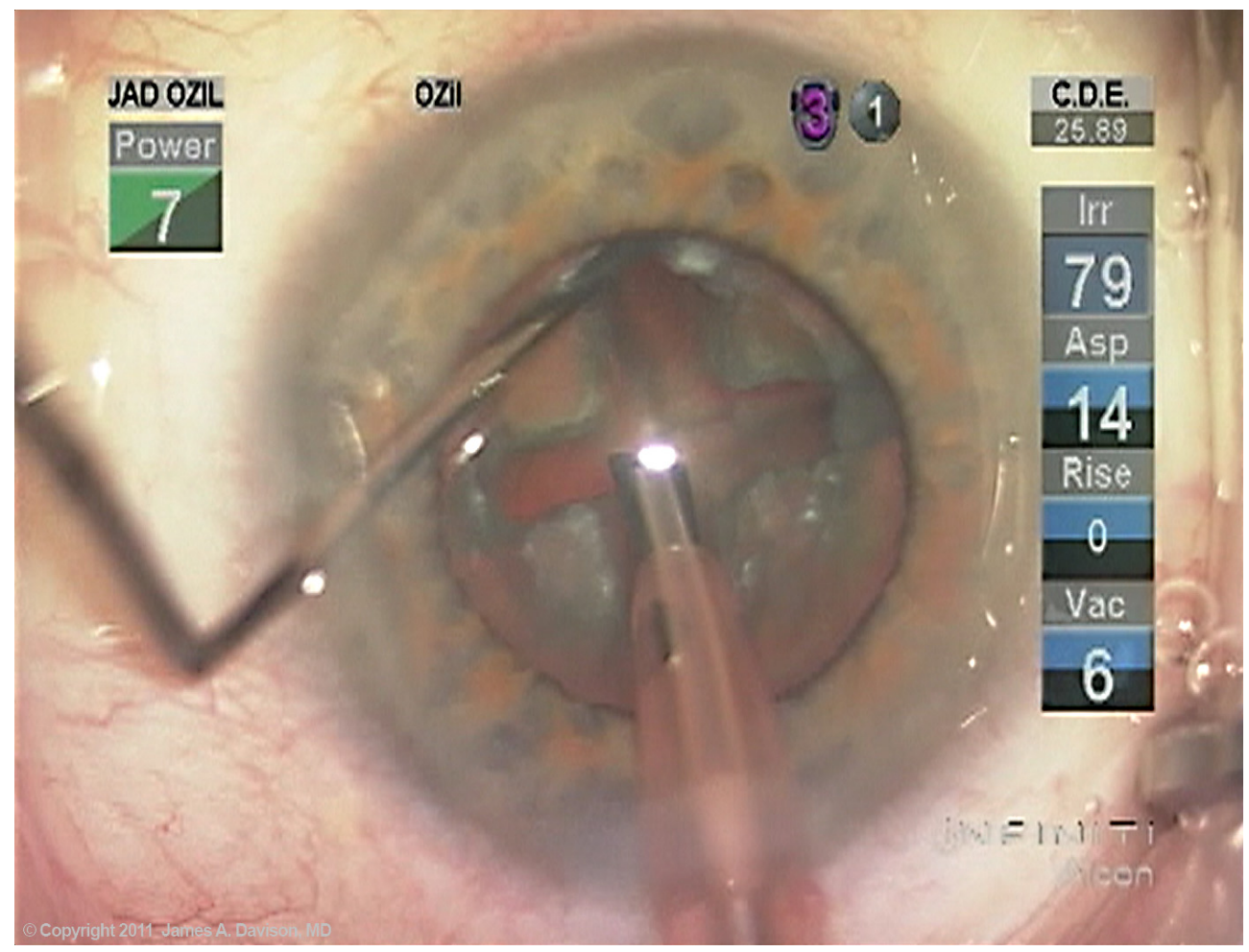

Fig. 8. Moderate width grooves have been sculpted through the nucleus creating 4 quadrants to be removed. 


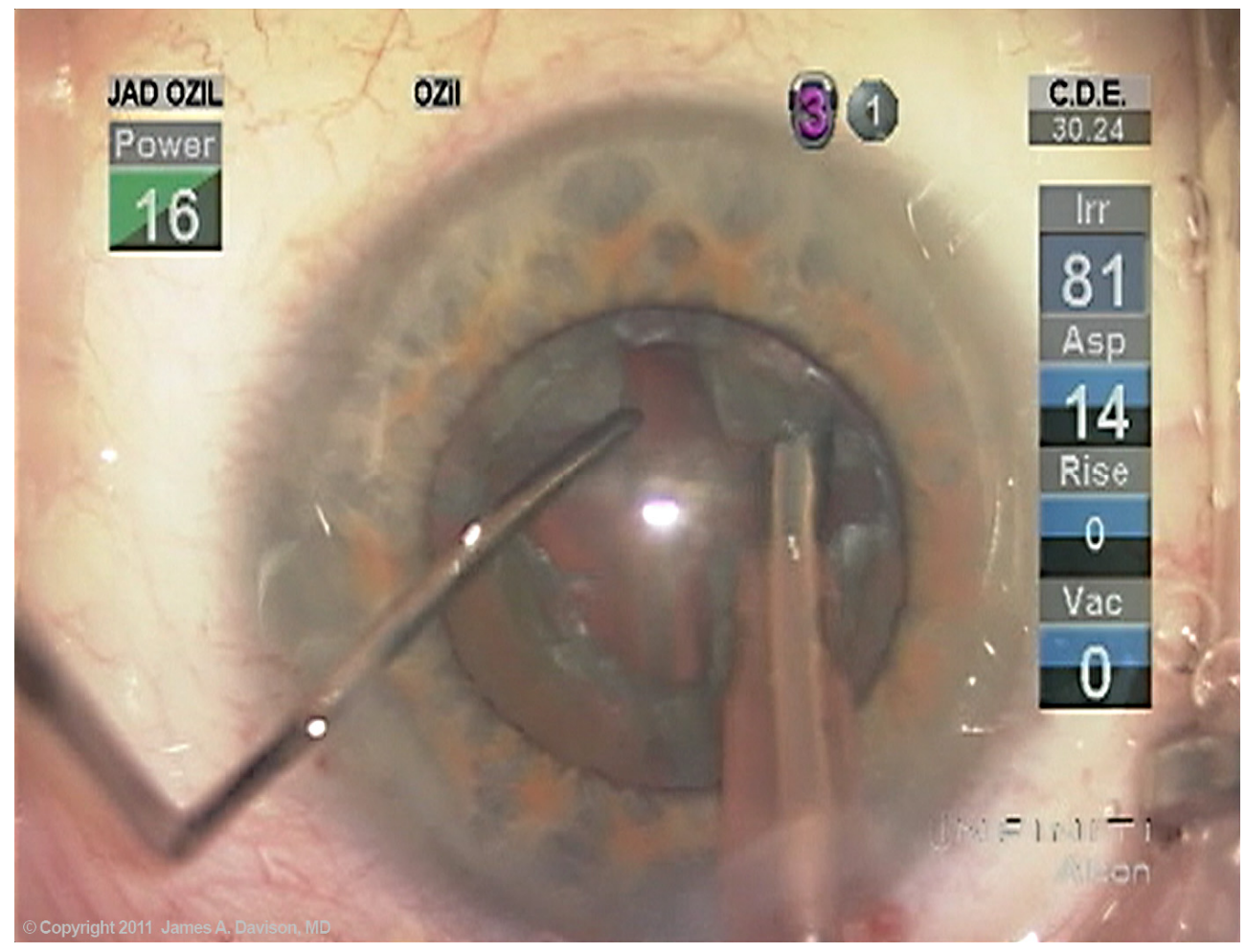

Fig. 9. The lower left quadrant has been de-bulked and the upper right one is about to be shaved in similar fashion. 


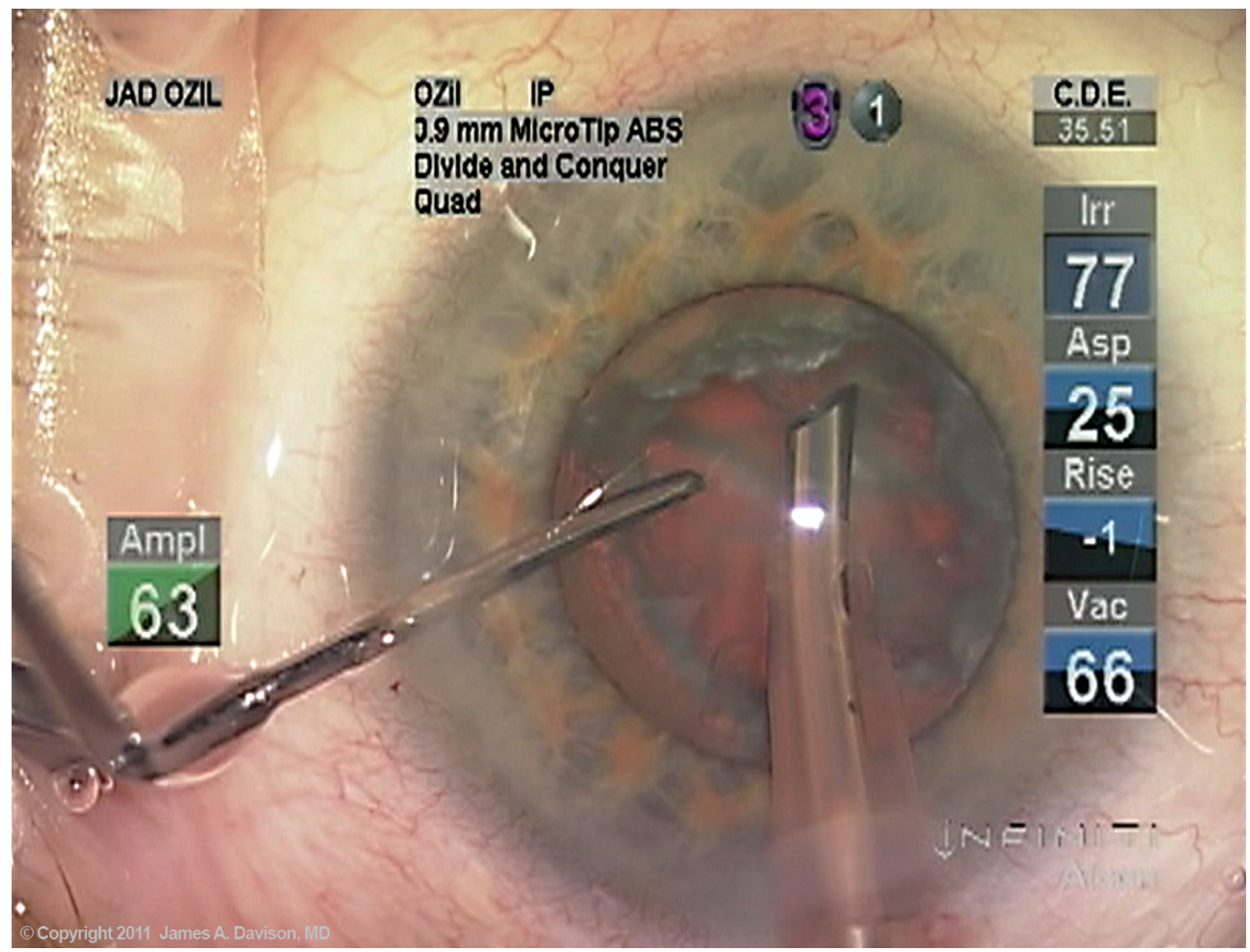

Fig. 10. All four quadrants have been debulked of the most firm central nuclear material leaving the more soft shell to be centralized and aspirated during quadrant removal.

In normal cases with normal size pupils, the quadrant removal settings are increased to a still fairly modest bottle height of $85 \mathrm{cms}$., vacuum of $400 \mathrm{~mm}$. Hg and AFR $35 \mathrm{cc}$. / min. maintaining a rise time of -1 but changing to a torsional motion with vacuum triggered interjected longitudinal ultrasonic tip motion (I.P., Alcon Surgical) (Vacuum Trigger $=90 \%$ of $400 \mathrm{~mm}$. $\mathrm{Hg}$, On Time $=7 \mathrm{msec}$, Longitudinal/Torsional ratio = 1.0). Continuous longitudinal ultrasound may be needed in extremely hard lenses in which I also use a rise time of -1 on the Infiniti to slow the rate of vacuum accumulation. In cases with borderline pupils, I reduce the aspiration flow rate to $25 \mathrm{cc} / \mathrm{min}$ in either IP or longitudinal to reduce flow, turbulence, iris fluttering, and miosis. This is extremely effective in maintaining pupil size. 


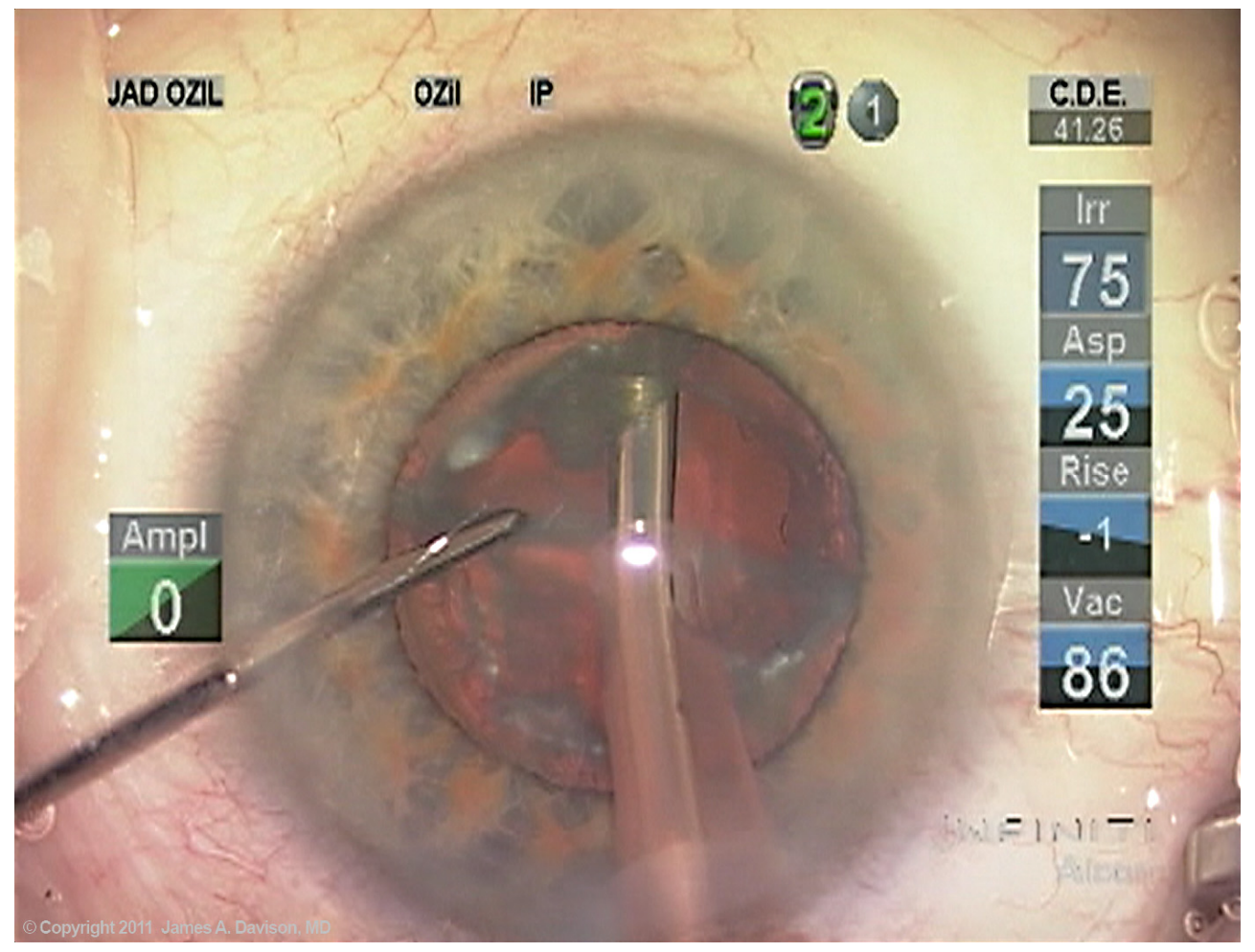

Fig. 11. The second quadrant is about to be engaged by the tip aperture which has been turned on its side for safer and easier access. 


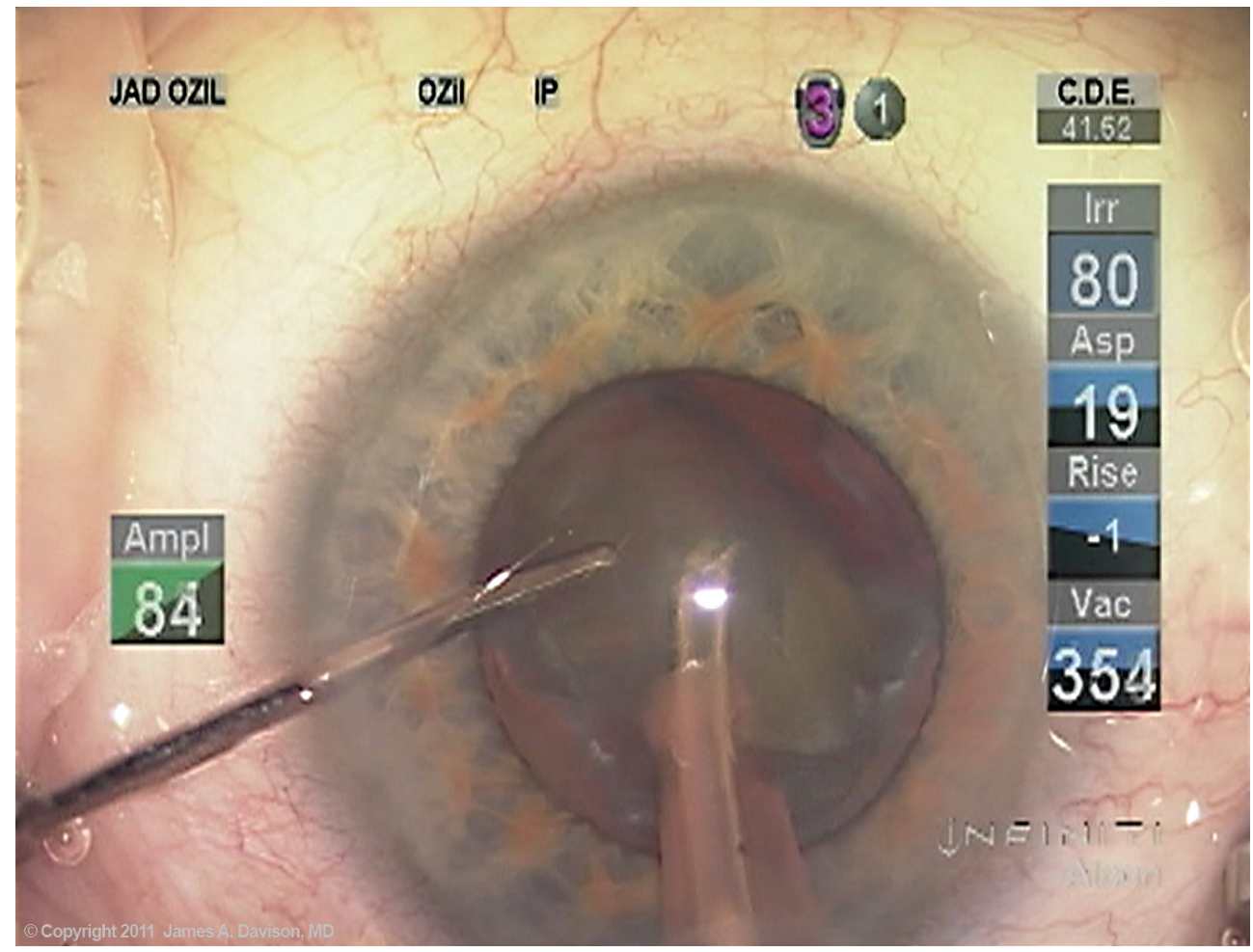

Fig. 12. The second quadrant has been centralized and the tip is oriented obliquely for best visualization and separation from the posterior capsule during its aspiration.

This reduction in flow creates a very quiet and stable anterior chamber and virtually eliminates any iris fluttering and consequent pupil size reduction. I'll usually shave the corners away during sculpting in these cases too, even if the nucleus is average firmness or soft so that the volume of material that is removed during this phase of surgery will be less. An additional measure which can be helpful is the injection of some additional Viscoat into the anterior chamber, especially preceding removal of the last quadrant. This protects the cornea, posterior capsule, and creates some additional dampening of hydraulic shock to the iris. To prevent thermal effects of the moving phacoemulsification tip at the incision, it's important to re-establish outflow by applying vacuum in foot position 2 prior to engaging phacoemulsification energy in foot position 3.

Modern machines like the Infiniti have minimized post-occlusion surge but there are still some shock waves transmitted to the iris by the quick oscillations of IOP secondary to the varying degrees of occlusion and pressure changes associated with aspiration of nuclear fragments in the quadrant removal mode. These pulsations are reduced by reducing the AFR to 25 while efficient removal of firm fragments is still possible using proportionally greater ultrasonic and vacuum contributions. It is most efficient to create a dynamic and continuous balance of ultrasonic energy, vacuum, and outflow during this phase of phacoemulsification. That way the extremes in pressure and flow are minimized and their 
secondary effects on the iris are minimized as well. In extremely hard nuclei, it may be sometimes necessary to use longitudinal phacoemulsification during the occlusion mode of quadrant removal to create this smooth balance.

\section{Prevention}

Preventing a floppy iris is much easier than managing it. By definition, an iris with or without a baseline flaccid tone only becomes floppy because of the turbulence it experiences once surgery has begun and BSS is flowing around it. Prevention is the key:

1. Use maximal pharmacologic dilation.

2. Create perfect incisions.

3. If the pupil size is small and there is too much iris surface area to start with, the pupil should be mechanically dilated i.e. the iris area should be made smaller. In normal circumstances, a $4.5 \mathrm{~mm}$. pupil and capsulorhexis diameter will be the minimum considered. Because of the affects of capsule contraction syndrome, it is particularly important to create at least a $5.0 \mathrm{~mm}$. diameter capsulorhexis in patients with pseudoexfoliation (Davison 1993).

4. Reduce nucleus bulk during sculpting with low vacuum and AFR

5. Use a longer rise time to reach maximum vacuum (-1 for me on the Infiniti).

6. A huge reduction in turbulence can be accomplished by using a low bottle height and aspiration flow rate during quadrant removal. Reducing the AFR to $25 \mathrm{cc} / \mathrm{min}$. and maintaining a BSS bottle height of $78 \mathrm{~cm}$. creates a quiet chamber, minimal turbulence, and virtually no iris fluttering and pupil diameter reduction.

7. If the iris does becomes floppy but the pupil size is still reasonable to continue phacoemulsification (depending on where in the process one is), additional Viscoat may tamponade turbulence effects on the iris, keep the pupil from becoming even smaller, and allow the case to be finished safely.

8. Whether the iris is floppy or not, if the pupil comes down to a level that makes the surgeon uncomfortable with the adequacy of visualization and the ability to execute surgical maneuvers, things can get difficult. That is, it's not so much about discovering if the iris will become floppy; it's more about the initial size of the pupil and attempting to maintain it by exercising smooth surgical technique through controlled hydrodynamics. If visualization becomes compromised because of pupil reduction or if iris is aspirated, iris retractors can still be placed at any stage during phacoemulsification, or even during the cortex aspiration stage if necessary. It's always better to stop and take a few moments to improve the situation and finish in an orderly fashion rather than to continue on an unnecessarily higher risk course.

\section{Opinions on floppy iris syndrome}

David Chang M.D. and John R. Campbell M. D. made an important discovery of an association of intraoperative complications caused by irises which appeared to be floppy during phacoemulsification in patients who were shown to be taking Tamsulosin (Flomax). (Chang 2005) Ever since then ophthalmic surgeons have been concerned about "Flomax patients" and iris related complications during cataract surgery. Even in television commercials for the product, patients are advised to reveal to their eye surgeons if they are on the medication. 
The author feels that the relationships between Flomax and floppy iris complications are in fact a statistical one. Almost all of the floppy iris cases of in their series were on Flomax but only about $60 \%$ of the patients who were on Flomax developed intraoperative floppy iris syndrome IFIS (Chang 2005). Mechanical stretching of the iris to make the pupil larger seemed to make the iris even more exposed to increased floppiness.

Even if patients are not on alpha 1 adrenergic antagonist, often patients with medium size pupils seem to have irises which suddenly flutter within the turbulence of BSS flow within the anterior chamber and behind the iris during phacoemulsification. Many times in these cases, the very first fluttering seems to initiate an almost immediate pupil constriction thus beginning the cascade to smaller and smaller pupil size because of increasing iris area and consequent increased capture of fluidic turbulence and more iris fluttering, just like a sailboat's larger jib catches more wind.

\section{References}

Chang DF, Campbell JR. Intraoperative floppy iris syndrome associated with tamsulosin. J Cataract Refract Surg. 2005; 31:664-673.

Chylack LT. The lens opacities classification system III. Arch Ophthalmol. June 1993; 111: 831-836.

Davison JA. Capsule Contraction Syndrome. J Cataract Refract Surg 1993; 19: 582-589.

Graether JM. Graether pupil expander for managing the small pupil during surgery. J Cataract Refract Surg 1996; 22:530-535.

Masket S, Belani S. Combined preoperative topical atropine sulfate $1 \%$ and intracameral nonpreserved epinephrine hydrochloride 1:2500 for management of intraoperative floppy-iris syndrome. J Cataract Refract Surg 2007; 33:580-582.

Shugar JK. Use of epinephrine for IFIS prophylaxis. J Cataract Refract Surg. July 2006; 32: 1074-1075. 